\title{
Determinantes del Rendimiento Académico de los Estudiantes de una Universidad Pública Ecuatoriana
}

\author{
Medina, Julio $^{1}$ (iD); Pinzón, Kathia ${ }^{2}$ iD ; Salazar-Méndez, Yasmín 1, * iD \\ ${ }^{I}$ Departamento de Economía Cuantitativa, Facultad de Ciencias, Escuela Politécnica Nacional, Quito, Ecuador \\ ${ }^{2}$ Centro de Estudios para el Desarrollo y Economía Aplicada (CEDEA), FLACSO-Ecuador
}

\begin{abstract}
Resumen: En el Ecuador, el sistema de educación superior históricamente se ha caracterizado por la sobrerrepresentación de los sectores más aventajados de la sociedad. En el año 2008, el Ecuador aprobó una nueva Constitución que incorporó nuevas directrices para la educación superior orientadas hacia la consecución de la igualdad de oportunidades. Con el fin de garantizar este principio, se implementó en 2012 el Sistema de Nivelación y Admisión (SNNA). En este contexto, el presente estudio tiene por objetivo analizar las características y el desempeño de los estudiantes de una facultad de una universidad pública ecuatoriana. Para esto, se aplicó una encuesta a un grupo de estudiantes y se estimó un modelo de regresión lineal múltiple. Los resultados sugieren, entre otras cosas, que aún existen sectores sociales con representación casi nula y que el desempeño académico universitario depende significativamente de variables asociadas a factores personales, familiares e institucionales.
\end{abstract}

Palabras clave: Desempeño académico, Modelo de regresión lineal múltiple, educación superior, Ecuador

\section{Determinants of Academic Performance of the Students of an Ecuadorian Public University}

\begin{abstract}
In Ecuador, the higher education system has been characterized by the overrepresentation of the most advantaged sectors of society. In 2008, Ecuador approved a new Constitution that incorporated new guidelines for higher education aimed at achieving equality of opportunities. In order to guarantee this principle, the so called Sistema Nacional de Nivelación y Admisión (SNNA) was implemented in 2012. In this context, the present study aims to analyze the characteristics and performance of students of a faculty of a public Ecuadorian university. For this, a survey was applied to a group of students and a multiple linear regression model was estimated. The results suggest, among other things, that there are still social sectors with almost zero representation and that university academic performance depends significantly on variables associated with personal, family and institutional factors.
\end{abstract}

Keywords: Academic performance, Multiple linear regression model, Higher education, Ecuador

\section{INTRODUCCIÓN}

La educación y, en particular, la educación superior (ES) cumplen un rol importante dentro de la consecución de los objetivos de desarrollo de un país. Las funciones que cumplen las instituciones de educación superior (IES), esencialmente la docencia y la investigación, no solamente garantizan a la población mejores oportunidades laborales, y con ello, mejores oportunidades económicas, sociales, etc., sino que también contribuyen al desarrollo productivo, empresarial, y económico (Valero \& Van Reenen, 2019). De esta forma, la ES constituye en la actualidad uno de los instrumentos principales con que cuentan los poderes públicos en su intento de asegurar el desarrollo de sus países, pues el gasto público destinado a esta finalidad es considerado una inversión de futuro (Baena, 1999).
A pesar de los indiscutibles beneficios de la ES, ésta, al igual que otros niveles de educación, suele presentar varias problemáticas consideradas desde diferentes perspectivas. Por ejemplo, considerando la región latinoamericana, Chiroleu (2011) menciona tres cuestiones que podrían considerarse medulares: (1) la expansión de la cobertura sin reducción de las desigualdades sociales ni inclusión plena en la ES, (2) el desarrollo de procesos de evaluación institucional sin mejoramiento efectivo de la calidad de los aprendizajes y, (3) el énfasis otorgado a la formación de profesionales dejando de lado el compromiso y la responsabilidad social de los universitarios.

A lo largo de la historia ecuatoriana, se ha podido evidenciar la coexistencia de las problemáticas mencionadas, sin embargo, a partir de la década de los 90, los análisis y

*yasmin.salazar@epn.edu.ec

Recibido: 20/07/2020

Aceptado: $23 / 03 / 2021$

Publicado: 31/05/2021

$10.33333 /$ rp.vol47n2.05

CC BY 4.0 
discusiones en torno a estos temas se han intensificado (Larrea, 2006). El resultado de la incorporación de los problemas de la ES ecuatoriana a la agenda política del país trajo como resultado algunas reformas que han intentado resolver tales problemáticas. Es así que, en el Ecuador, han sido varias las políticas implementadas en referencia a la temática de la ES, especialmente durante el último gobierno (que gobernó entre el año 2007 y 2017).

En vista de que el análisis de los determinantes del rendimiento estudiantil universitario es una de las claves para la proposición de medidas tendientes al mejoramiento de la educación y al desarrollo del país, luego de varios años de la implementación del Sistema Nacional de Nivelación y Admisión (SNNA), se realiza la presente investigación que tiene por objetivo identificar las características socioeconómicas y el desempeño de los estudiantes de una de las facultades más numerosas de una de las mejores universidades públicas ecuatorianas. Para esto, a partir de datos primarios, se realiza un análisis de estadística descriptiva para obtener la caracterización socioeconómica de los estudiantes y mediante la estimación de un modelo de regresión lineal múltiple se determinan los factores que inciden en el desempeño académico de los estudiantes. Lo que resta del artículo está organizado de la siguiente manera. En la sección 2 se describe el sistema de ES ecuatoriano, mientras que en la sección 3 se presenta la revisión de literatura referente a los factores que inciden en el desempeño académico estudiantil. Los datos y la metodología de análisis aparecen en la sección 4 y los resultados obtenidos se muestran en la sección 5. Finalmente, en la sección 6 se señalan las conclusiones de la investigación.

\section{EL SISTEMA DE EDUCACIÓN SUPERIOR ECUATORIANO ACTUAL}

En el 2008, el Ecuador aprobó su Constitución actual e incorporó nuevas directrices para la ES encaminadas a la consecución de la igualdad de oportunidades ${ }^{2}$. En la Carta Magna, se establece la responsabilidad del Estado como proveedor de la educación pública (Art. 26) y se menciona que esta constituye un área prioritaria de la política pública y de la inversión estatal, garantía de la igualdad e inclusión social, y que es una condición indispensable para el buen vivir; se manifiesta, además, explícitamente, que el sistema de ES se regirá, entre otros principios, por la igualdad de oportunidades, y que el ingreso a las instituciones públicas de educación superior se regulará a través de un sistema de nivelación y admisión definido por la ley.

Con el objetivo de garantizar la operatividad en la implementación de estos principios en la ES, la Constitución de 2008 también estableció la necesidad de dictar una nueva Ley Orgánica de Educación Superior (LOES), misma que tiene como objetivos: regular el sistema de ES del Ecuador y garantizar el derecho a una ES de calidad y que propenda a la excelencia, al acceso universal, permanencia, movilidad y egreso sin discriminación alguna. Dentro de esta normativa,

\footnotetext{
${ }^{2}$ En el artículo 71 del Capítulo I del Título IV de la Ley Orgánica de Educación Superior (LOES) de Ecuador, se manifiesta que el principio de igualdad de oportunidades consiste en garantizar a todos los actores del Sistema de Educación Superior las mismas posibilidades en el acceso,
}

entre otras cosas, se estipula que, para garantizar el principio de igualdad de oportunidades, mérito y capacidad, el ingreso a las IES públicas estará regulado a través del SNNA, al que se someterán todos los estudiantes aspirantes.

Mediante el SNNA, entonces, el Estado, representado en esta instancia por la Secretaría de Educación Superior, Ciencia, Tecnología e Innovación (SENESCYT), administra y coordina el proceso de ingreso de los aspirantes a las IES públicas. Para tal efecto dicha institución implementó, por una parte, el Examen Nacional para la Educación Superior (ENES) con el cual se evaluarían las habilidades de los aspirantes y, por otra, distintas modalidades de cursos de nivelación e instrumentos de evaluación, con la finalidad de garantizar una inserción exitosa de los aspirantes al Sistema de ES (SENESCYT, 2012). Este sistema comenzó a aplicarse a partir del año 2012.

No obstante, se han realizado modificaciones a dicho sistema. Inicialmente, los aspirantes debían rendir el ENES y esperar la asignación de un cupo en una de las carreras incluidas en la lista de las preferencias del candidato. Esta asignación era realizada por la SENESCYT considerando el puntaje alcanzado por el candidato y el número de plazas ofertadas por cada IES. El puntaje mínimo requerido para ingresar a estudios de tercer nivel en instituciones públicas se estableció en 600 sobre 1000 puntos. En febrero de 2017, se fusionaron las pruebas de conocimientos para estudiantes de secundaria "Ser Bachiller", con el ENES, y se eliminaron los puntajes mínimos para ingresar a una carrera. Así, la aprobación del examen "Ser Bachiller", además de otorgar el título de bachillerato, permitiría el ingreso a la ES pública.

La creación de la Ley Orgánica de Educación Superior (LOES) en octubre de 2010, y la creación y ejecución del SNNA en el 2012, ciertamente constituyen algunas de las modificaciones más importantes que se han realizado al sistema de educación superior ecuatoriano durante los últimos años. A pesar de esto, el que estas políticas hayan promovido un "mejoramiento" en el sistema de ES, es aún algo incierto. Son varias las observaciones, positivas y negativas, y los cuestionamientos que se han hecho a las modificaciones mencionadas y a los efectos que éstas han tenido en el sistema de ES. Por ejemplo, existen sectores que pregonan un incremento en la representatividad de grupos históricamente excluidos indígenas, discapacitados, grupos de bajos ingresos- en las IES. Por otro lado, existen quienes sugieren que los grupos subrepresentados no han sido beneficiados con el proceso y aún permanecen excluidos de las IES públicas, así como también existen quienes afirman que fallas en el SNNA son las causantes del bajo rendimiento académico de los estudiantes universitarios.

Con referencia a aquellos cuestionamientos, se genera la dubitativa de si efectivamente la política sobre ES que se aplicó (la contemplación de gratuidad de la educación en la LOES, la consecuente implementación del SNNA, etc.) durante los últimos años está cumpliendo con sus objetivos primordiales. Varios autores---con base en investigaciones 
realizadas en otros países---coinciden, por ejemplo, en que la política de gratuidad de la ES bien podría favorecer el acceso de los jóvenes provenientes de los estratos socioeconómicos superiores, generando mayores oportunidades de acceso a estudiantes de hogares con mayor ingreso y mayor nivel educativo (Jerrim, Chmielewski \& Parker, 2015). Por otra parte, este mismo efecto sería generado por los sistemas de admisión universitaria que se basan solamente en evaluaciones cognitivas, restando así oportunidades para aspirantes de familias con bajos ingresos (Mountford-Zimdars, Moore y Graham, 2016).

Respecto a la situación de la ES en el Ecuador, Larrea (2006), por ejemplo, menciona en su investigación que, especialmente en el período entre 1990 y 2006, las políticas sociales aplicadas en el país habían promovido la inversión pública en los niveles primario y secundario, en detrimento de la instrucción superior, lo cual había ocasionado altos niveles de desigualdad en temas de escolaridad. Por otro lado, y en relación a las características del acceso universitario, según Ponce y Carrasco (2016), entre 1999 y 2014 la tasa bruta de matrícula universitaria incrementó de $20.2 \%$ a $29.6 \%$ y, a diferencia de lo sucedido en el período 1999-2006, entre 2007 y 2014 tal incremento no se concentró en los quintiles más altos de la población, sino en los grupos poblacionales de la zona urbana, de la Sierra y de mujeres. Adicionalmente, los autores mencionan que, entre 2006 y el 2014, la tasa bruta de matrícula incrementó mayormente entre los blancos, seguido por los mestizos, los afroecuatorianos y los indígenas, pero que también incrementó significativamente para estudiantes pertenecientes al quintil de ingresos más alto; a pesar de esto, mientras en 2006 los primeros cinco deciles más pobres y el decil más rico participaban con el $12 \%$ y el $32 \%$ de la matrícula universitaria, respectivamente, en el 2014 dicha participación pasó a ser del $18 \%$ y el $25 \%$, respectivamente.

Por otra parte, con relación al rendimiento académico (RA) estudiantil universitario en el Ecuador y los factores que influyen en este, y particularmente, factores institucionales en los que pueden verse inmersas las políticas aplicadas a la ES, según nuestro conocimiento, no se ha llevado a cabo investigación formal alguna; es decir la evidencia empírica con respecto a este tema es incipiente. Esto, a pesar de que el análisis de los factores, tanto sociales, como económicos, institucionales, y demás, que influyen en el rendimiento académico estudiantil universitario es importante, puesto que permite la identificación de mecanismos orientados hacia su mejoramiento y, consecuentemente, hacia la formación de mejores profesionales y posteriormente hacia el desarrollo del país.

\section{FACTORES QUE INCIDEN EN EL DESEMPEÑO ACADÉMICO ESTUDIANTIL}

Una cantidad considerable de literatura se ha desarrollado alrededor del mundo respecto a los factores que influyen en el desempeño académico estudiantil en los diferentes niveles de educación. En relación a la ES, en particular, se han llevado a cabo varios análisis sobre esta temática desde diferentes perspectivas: el punto de vista psicológico (Garbanzo, 2007), el punto de vista estadístico (Shahzadi \& Ahmad, 2011)
(Sulemana, Bin, \& Majid, 2014) y hasta desde la perspectiva organizacional (Fonseca \& García, 2016), entre otras.

Con respecto al primer aspecto, Garbanzo (2007), Erazo (2012), Lerner (2012) y Fonseca y García (2016), realizan análisis teóricos que dan cuenta de los factores que se conjugan en la vida de un estudiante universitario y que, consecuentemente, pueden afectar a su rendimiento académico. Los resultados relevantes de tales análisis se pueden resumir en lo expuesto por Garbanzo (2007) en su investigación. Según esta autora, el rendimiento académico universitario es efectivamente el resultado de un proceso en el que intervienen de forma interrelacionada los siguientes factores: personales, sociales e institucionales.

Dentro de los factores personales, Garbanzo menciona a la competencia cognitiva, la motivación, las condiciones cognitivas, el autoconcepto académico, la autoeficacia percibida, el bienestar psicológico, la satisfacción con los estudios, la asistencia a clases, la inteligencia, las aptitudes, el sexo, la formación académica previa, y la nota de acceso a la universidad. Respecto a los factores sociales, la autora sugiere que son las diferencias sociales, el entorno familiar, el nivel educativo de los progenitores o responsables del estudiante, el nivel educativo de la madre, el contexto socioeconómico y demás variables demográficas, los determinantes del desempeño académico. Finalmente, como factores institucionales, que son los relacionados con la universidad en sí, en la investigación se menciona a la elección de los estudios según el interés del estudiante, la complejidad de los estudios, las condiciones institucionales, los servicios institucionales de apoyo, el ambiente estudiantil, la relación estudiante-profesor, y las pruebas de ingreso a la carrera.

En cuanto a investigaciones aplicadas, orientadas a la búsqueda de evidencia empírica del desempeño académico de estudiantes universitarios, también existen varios resultados importantes. Por ejemplo, Guzmán (2012) estima un modelo de regresión múltiple considerando variables relacionadas con la información académica y personal de los estudiantes de una universidad privada de México, y concluye que los factores que influyen significativamente en el RA universitario son el desempeño académico en la secundaria, la edad, la recepción de alguna beca, la calificación de ingreso a la universidad y la cantidad de materias reprobadas (únicamente la edad y la cantidad de materias reprobadas influyen negativamente). En la investigación de Li y Carroll (2017), son la edad y la cantidad de materias aprobadas variables significativas y positivamente influyentes en el RA. Además, en esta última investigación, para la cual se utilizó información referente a estudiantes de universidades públicas y privadas de Australia, se concluyó que el género es un determinante del RA únicamente durante los últimos semestres y que, asimismo, la modalidad de estudio, presencial o a distancia, influye únicamente durante los primeros semestres. Adicionalmente, los autores concluyen que, tanto en los primeros como en los últimos semestres, la pertenencia a determinados grupos vulnerables (específicamente minorías étnicas, personas cuyo idioma es distinto a su idioma de estudios, personas discapacitadas y personas de bajos recursos económicos) es una variable que incide significativamente en el RA. Por otro lado, la ubicación del domicilio en zonas remotas o 
inaccesibles sería una condición influyente únicamente para estudiantes en los últimos semestres, mientras que la característica de ser el primero de la familia en cursar estudios de nivel superior es una variable determinante del RA durante los primeros semestres.

Finalmente, se han realizado también varios análisis que utilizan la estadística (netamente) descriptiva para intentar aproximarse hacia una caracterización del rendimiento de estudiantes universitarios. Por ejemplo, Martínez et al. (2011) quienes analizaron a un grupo de estudiantes de Venezuela, observaron que los estudiantes presentaban mayor RA promedio a medida que avanzaba su edad; por otro lado, estudiantes solteros presentaron mayores niveles de RA promedio que estudiantes casados. Ramírez (2014), autor que analizó con un enfoque descriptivo la situación de estudiantes colombianos y las características de su rendimiento por género y otros factores, observó que las estudiantes solteras obtuvieron un mayor RA promedio que el de las no-solteras (este hecho, sin embargo, no se evidenció para el caso de los hombres), y además, que los estudiantes con mayores edades presentaron un desempeño académico inferior a quienes eran menores (esto, sin embargo, no se verificó para el caso de las mujeres). Finalmente, en un contexto geográfico y cultural lejano al latinoamericano, Li y Carroll (2017), basados también en un análisis descriptivo, reportaron que, en el caso de los estudiantes universitarios australianos, quienes pertenecían a minorías étnicas tuvieron un desempeño académico menor, al ser comparados, con los grupos étnicos mayoritarios. Un factor en el que coincide la dirección de los resultados de las tres investigaciones de enfoque descriptivo es la renta: los estudiantes pertenecientes a los estratos económicos más altos presentaron mejor desempeño (en promedio) con respecto a los estudiantes de renta baja.

\section{DATOS Y METODOLOGÍA}

\subsection{Datos}

Los datos para describir al grupo de estudiantes y para determinar los factores que inciden en su desempeño académico fueron obtenidos a partir de una encuesta, que fue aplicada a los estudiantes de una de las facultades más numerosas de una universidad pública ecuatoriana. Las carreras que ofrece dicha facultad son: física (FI), ingeniería en ciencias económicas (IC), ingeniería matemática (IM) y matemática (MA).

Se realizó un muestreo aleatorio, considerando una población total finita (cantidad total de estudiantes de la facultad) de tamaño $N=777$, un límite máximo para el error de estimación de $\varepsilon=0.05$, un nivel de confianza de $95 \%$, y una desviación estándar (para la variable IRA, con base en la información poblacional existente previamente en registros administrativos) de $\hat{\sigma}=8.586$. Así se determinó, de acuerdo con la expresión $\varepsilon \approx z_{\alpha / 2} \sqrt{\frac{N-n}{N-1}} \frac{\widehat{\sigma}}{n}$, que $n$ es tal que

\footnotetext{
${ }^{3}$ Según la Ley Orgánica de Educación Intercultural de Ecuador, expedida en 2011, son instituciones educativas fiscomisionales aquellas cuyos promotores son congregaciones, órdenes o cualquiera otra denominación confesional o laica; son de carácter religioso o laica, de derecho privado y sin fines de lucro, y cuentan con financiamiento total o parcial del Estado, con la
}

$0.05 \approx 1.95996 \sqrt{\frac{777-n}{776}} \frac{8.586}{n}, \mathrm{y} \quad n \approx 271.61$. El tamaño muestral es, entonces, de 272 estudiantes (Scheaffer, Mendenhall \& Ott, 2006).

Con la finalidad de minimizar los riesgos de no obtención de la cantidad de información necesaria para el análisis (272 observaciones), para la realización de las encuestas se convocó aleatoriamente a un total de 330 estudiantes. Con la información obtenida, luego de realizar una limpieza de datos (eliminación de casos de no respuesta, y casos de respuestas no válidas como ingresos familiares o edades iguales a 0 ), se obtuvo un total de 284 observaciones. Dichas observaciones se distribuyen, por carreras, de la siguiente forma: $19 \%$ (FI), $43 \%$ (IC), 26\% (IM) y $10 \%$ (MA).

Con respecto a la encuesta, esta tuvo por objetivo recabar información relacionada con los determinantes propuestos por Garbanzo (2007). En ese sentido, las variables de las cuales se recabó información y su respectiva categoría son las siguientes:

- Factores personales: edad, género, estado civil, etnia, discapacidad, satisfacción con la carrera, número de reprobaciones, tipo de colegio (público/privado/fiscomisional ${ }^{3}$ ), horas de estudio semanales, tipo de bachillerato, y rendimiento académico (medido por el denominado Índice de Rendimiento Académico "IRA", que es el indicador de RA utilizado en la universidad analizada).

- Factores institucionales: proceso de elección de la carrera, tipo de carrera, sistema de ingreso.

- Factores sociales: nivel de educación de los padres, ingreso familiar per cápita, área de residencia.

\subsection{Metodología}

Para determinar los factores que afectan en el desempeño de los estudiantes de la facultad analizada, se estima un modelo de regresión lineal múltiple que responde a la siguiente especificación econométrica:

$\ln (I R A)_{i}=\boldsymbol{\beta}_{\mathbf{0}}+F P_{i} \boldsymbol{\beta}_{\mathbf{1}}+F I_{i} \boldsymbol{\beta}_{\mathbf{2}}+F S_{i} \boldsymbol{\beta}_{\mathbf{3}}+u_{i}$

Donde:

$\ln (I R A)_{i}$ es el logaritmo del IRA del individuo i.

$F P_{i}$ es un vector (fila) de factores personales del individuo i. Este vector tiene como coordenadas los valores (para el individuo) de las siguientes variables. Edad, que es una variable numérica. Créditos aprobados, que es una variable numérica. Tipo de bachillerato de procedencia, que es una variable categórica (tres categorías): "Bachillerato en ciencias", "Bachillerato técnico", "BGU"), con "Bachillerato en ciencias" como categoría base. Reprobó, que es una variable categórica, con no ha reprobado como categoría base.

condición de que se cumpla el principio de gratuidad, igualdad de oportunidades para el acceso y permanencia, rendición de cuentas de sus resultados educativos y manejo de los recursos y el respeto a la libertad de credo de las familias. 
$F I_{i}$ es un vector (fila) de factores institucionales del individuo i. Este vector tiene como coordenadas los valores (para el individuo) de las siguientes variables. Carrera, que es una variable categórica (4 categorías: "IC", "FI", "IM", "MA"), con "IC" como categoría base.

$F S_{i}$ es un vector (fila) de factores sociales del individuo i. Este vector tiene como coordenadas los valores (para el individuo) de las siguientes variables. Quintil de ingreso, que es una variable categórica (5 categorías), con el quintil 1 de base. Madre con educación superior, que es una variable dicotómica, con "madre no tiene educación superior" como categoría base. Padre con educación superior, que es una variable dicotómica, con "padre no tiene educación superior" como categoría base.

Finalmente, $u_{i}$ es el término de error. El modelo clásico de regresión lineal supone que cada $u_{i}$ está normalmente distribuido con: $E\left(u_{i}\right)=0 ; \operatorname{Var}\left(u_{i}\right) \sigma^{2}$ y $\operatorname{cov}\left(u_{i}, u_{j}\right)=$ $0, \forall i \neq j$. Estos supuestos pueden resumirse en $u_{i} \sim N\left(0, \sigma^{2}\right) \forall i$ (Wooldridge, 2012).

Todas las variables dicotómicas toman el valor de 0 en los casos en que el individuo pertenece a la categoría "base", y el valor de 1 en caso contrario. Por otro lado, la inclusión de una variable categórica de " $k$ " categorías, con $k \geq 3$, como es conocido, constituye la inclusión de $k-1$ variables dicotómicas, cada una de las cuales compara la categoría "base" con cada una de las $k-1$ categorías restantes. Así, por ejemplo, una variable categórica de 3 categorías ("a", "b" y "c"), con "a" como categoría base, se representará en el modelo mediante 2 variables dicotómicas: una que toma el valor de 0 en caso de "a" y de 1 en caso de "b", y una que toma el valor de 0 en caso de "a" y de 1 en caso de "c".

Después de la estimación, se realizan los análisis respectivos. Según Greene (2004), para el análisis de correcta especificación de un modelo de regresión lineal se deben observar esencialmente los siguientes aspectos: homoscedasticidad (test de Breusch Pagan), variable omitida (test RESET), multicolinealidad (evaluación del factor de inflación de la varianza, VIF).

Para la realización de todos los análisis, tanto de tipo descriptivo, así como la estimación del modelo aquí descrito, se utiliza el software estadístico STATA (Versión 16).

\section{RESULTADOS Y DISCUSIÓN}

\subsection{Caracterización socioeconómica de los estudiantes}

En esta subsección se presentan los resultados del análisis de estadística descriptiva de la información recabada en la encuesta.

\subsubsection{Rendimiento Académico}

Para analizar el rendimiento académico de los estudiantes se consideró el IRA. El IRA se calcula ponderando todas las notas de las materias aprobadas y reprobadas por un estudiante; en este cálculo no se incluyen las notas de materias anuladas o canceladas. Respecto al comportamiento del rendimiento académico por carrera, se observa que este es mayor en la carrera de IC (IRA promedio de 26.1), seguido consecutivamente de las carreras de IM, MA y FI (con IRA promedio de $24.7,22.6$ y 22.4 , respectivamente).

Para analizar el comportamiento del rendimiento académico, se han conformado grupos en torno a los resultados en investigaciones anteriores respecto a esta temática. En la Tabla 1 se muestran los resultados del IRA promedio para determinados grupos cuyo análisis es de especial interés en la literatura.

Tabla 1. IRA promedio por grupos de interés

\begin{tabular}{lllll}
\hline Categorías/Grupos & FI & IC & IM & MA \\
\hline \hline Grupos por edad & & & & \\
Grupo 1 & 10.6 & 24.2 & 19.8 & 19.1 \\
Grupo 2 & 22 & 26.5 & 24.1 & 22.3 \\
\hline Grupos por género & & & & \\
Femenino & 19.6 & 25.4 & 22.7 & 20.3 \\
Masculino & 18.2 & 26 & 22.3 & 20.6 \\
\hline Grupos por tipo de institución secundaria & & & & \\
Institución Fiscal & 21.4 & 24.6 & 21.9 & 21 \\
Institución Particular & 19.1 & 27.1 & 24 & 18.8 \\
\hline
\end{tabular}

Respecto al desempeño académico por grupo de edad, se consideraron dos grupos: uno conformado por estudiantes de hasta 21 años (Grupo 1), y otro conformado por estudiantes mayores de 21 años (Grupo 2). Se ha realizado esta diferenciación puesto que es alrededor de esta edad que los estudiantes superan los tres primeros semestres de su carrera (en los cuales se conoce que existen altos índices de repetición). Los resultados muestran que en todas las carreras el rendimiento promedio del Grupo 2 supera al del Grupo 1. Este resultado concuerda con lo evidenciado en la investigación de Martínez et al. (2011) y según los autores, este hecho podría deberse a que los primeros años de estudios universitarios representan un cambio drástico en la modalidad de aprendizaje de los estudiantes, motivo por el cual su rendimiento puede verse afectado.

De acuerdo al género, los resultados muestran que los IRA promedio de hombres y mujeres son bastante similares en todas las carreras. Finalmente, en las carreras de IC e IM los estudiantes provenientes de instituciones educativas particulares poseen los mayores IRA, mientras que en FI y MA los mayores índices corresponden a estudiantes procedentes de colegios fiscales.

Tabla 2. IRA promedio por carrera, por quintil de ingreso y

\begin{tabular}{|c|c|c|c|c|c|c|c|c|c|c|c|c|}
\hline \multirow{2}{*}{ Quintil } & \multicolumn{3}{|c|}{ FI } & \multicolumn{3}{|c|}{ IC } & \multicolumn{3}{|c|}{ IM } & \multicolumn{3}{|c|}{ MA } \\
\hline & $\mathbf{F}$ & M & Total & $\mathbf{F}$ & M & Total & $\mathbf{F}$ & M & Total & $\mathbf{F}$ & M & Total \\
\hline 1 & 19.3 & 14.8 & 16.8 & 23.6 & 26.14 & 24.3 & 14.8 & 21.8 & 19.3 & 16 & 13.8 & 14.8 \\
\hline 2 & 17.3 & 17 & 17.1 & 23.6 & 29.6 & 25.2 & 25.1 & 22.5 & 23.8 & -- & 25.5 & 25.5 \\
\hline 3 & 22 & 20 & 20.7 & 28.1 & 26.5 & 27.3 & 26.5 & 22 & 23.3 & 23.5 & 18.8 & 20.1 \\
\hline 4 & 20.4 & 18.3 & 19.6 & 26.2 & 26.1 & 26.2 & 23.3 & 21.3 & 22.1 & 31 & 25.7 & 27 \\
\hline 5 & 18.8 & 22 & 20.7 & 26.6 & 23.4 & 25.3 & 17.7 & 24.3 & 22.8 & 20 & 20.5 & 20.4 \\
\hline
\end{tabular}

La caracterización de los estudiantes considerando el quintil de renta, el género y la carrera se muestra en la Tabla 2. Se evidencia que los estudiantes de los quintiles más altos de ingreso no necesariamente muestran los mejores IRA. De hecho, solamente en el grupo de hombres de las carreras de física y de ingeniería matemática, los IRA promedio más altos corresponden a estudiantes del quintil 5. Por otra parte, también se puede observar, en casi todos los grupos, con 
excepción del grupo de hombres de IC e IM, que los menores índices de RA corresponden a estudiantes ubicados en el quintil 1 y 2. En la mayor parte de los grupos analizados, los mejores IRA promedio corresponden a estudiantes del quintil 3.

\subsubsection{Características personales, sociales e institucionales}

En lo que respecta a las características personales de los entrevistados, la muestra está formada por un $46 \%$ de personas de género femenino y $54 \%$ de género masculino. Los hombres son mayoría en las carreras de física, matemática e ingeniería matemática, con representación de hombres en aproximadamente un $60 \%, 75 \%$ y $60 \%$, respectivamente, mientras que en IC hay presencia mayoritaria de mujeres (60\%). La carrera de IC es la única carrera con representación mayoritaria de mujeres. Estos resultados sugieren que, en concordancia con la literatura existente las mujeres prefieren mayormente carreras relacionadas con ciencias sociales y de la vida (Leyton, Sánchez y Ugalde, 2010).

La etnia predominante es de quienes se autoidentificaron como mestizos (96\%). Las minorías étnicas tienen una participación que no sobrepasa el 2\%. El estado civil del $98 \%$ de los encuestados es soltero. Finalmente, la edad de los entrevistados oscila entre los 18 y 32 años.

Observando los datos asociados con las características académicas, de la muestra se concluye que el $52 \%$ de los encuestados proviene de una institución secundaria fiscal, seguidos por los que estudiaron en un colegio particular (35\%) y fiscomisional (13\%).

Cuando se indagó a los participantes de la encuesta sobre la prioridad que dieron a la carrera que actualmente están estudiando en el sistema de elección de carrera del SNNA, el $64 \%$ respondió que la carrera corresponde a la primera opción y el $16 \%$ a la segunda opción. El porcentaje restante corresponde a los estudiantes que colocaron a la materia como tercera opción $(8 \%)$ y los estudiantes que ingresaron antes de este sistema de elección de carrera (12\%). Es decir, a partir de estas cifras se puede inferir que la mayoría de estudiantes estaría cursando una carrera que pertenece a su lista de preferencias. Esta cifra parece estar en concordancia con el nivel de satisfacción de los estudiantes con la carrera elegida y cuyos resultados se muestran en la Figura 1; más del $60 \%$ de estudiantes respondieron que su nivel de satisfacción es alto o muy alto para calificar el nivel de satisfacción con la carrera que están estudiando.

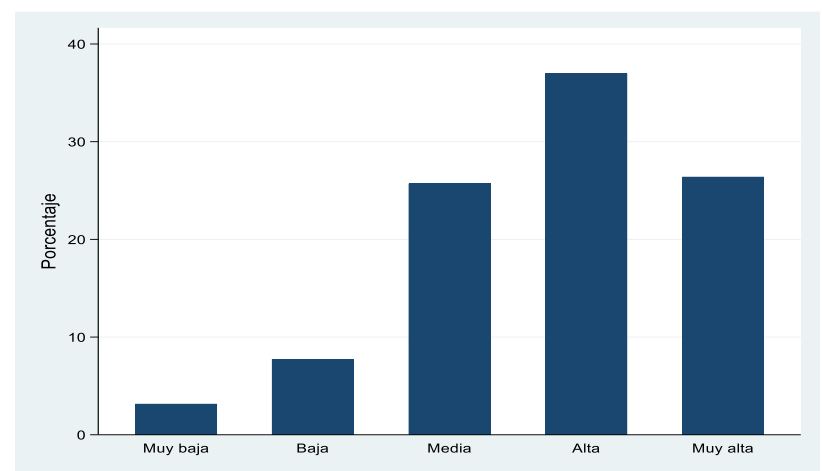

Figura 1. Nivel de satisfacción de los encuestados con la carrera

A nivel de carreras, MA e IM muestran los mayores niveles de satisfacción (ver Tabla 3).

Tabla 3. Nivel de satisfacción con la carrera elegida

\begin{tabular}{ccccc}
$\begin{array}{c}\text { Nivel de } \\
\text { satisfacción }\end{array}$ & FI & IC & IM & MA \\
\hline 1 & $2 \%$ & $6 \%$ & $3 \%$ & $0 \%$ \\
2 & $14 \%$ & $8 \%$ & $4 \%$ & $9 \%$ \\
3 & $25 \%$ & $30 \%$ & $25 \%$ & $21 \%$ \\
4 & $35 \%$ & $36 \%$ & $39 \%$ & $33 \%$ \\
5 & $24 \%$ & $20 \%$ & $29 \%$ & $37 \%$ \\
\hline
\end{tabular}

A pesar de las cifras mostradas en la Tabla 3, que sugieren un alto nivel de satisfacción con la carrera, llama la atención el porcentaje de estudiantes que han reprobado alguna asignatura: $81 \%$ de los encuestados reportaron haber reprobado una disciplina alguna vez en su vida estudiantil. Estas reprobaciones no parecen ser exclusividad de ninguna carrera pues como se muestra en la Figura 2, la alta tasa de reprobaciones se presenta en todas las carreras de la facultad analizada. La gráfica contiene las respuestas de la pregunta: ¿ha reprobado alguna materia?

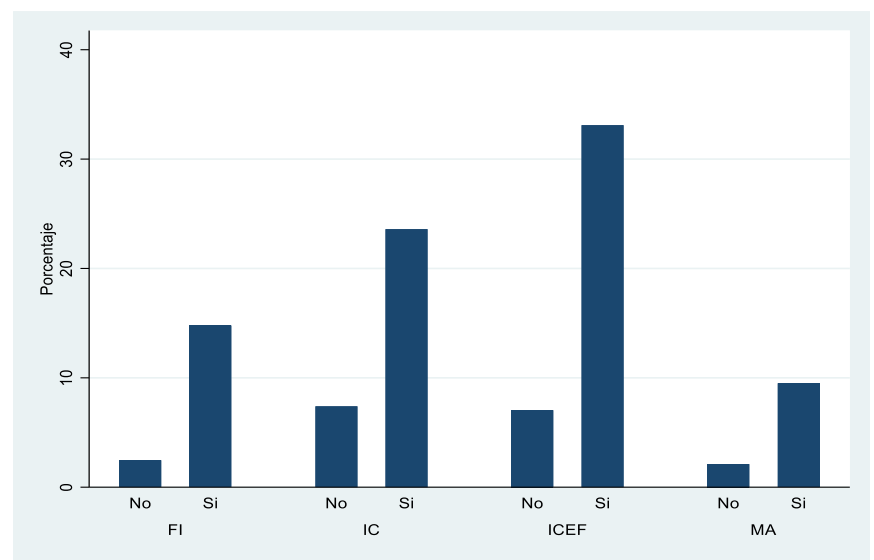

Figura 2. Reprobación de materias de los encuestados

Con relación a las horas de estudio semanales, estas presentan una gran dispersión; pues mientras el promedio es de 16 horas, existen estudiantes que reportan una hora de estudio a la semana $\mathrm{u}$ otros que manifiestan que este valor corresponde a 65 horas. Los valores bajos en cuanto a horas de estudio semanales pueden ser asociados al hecho de que el $15 \%$ de estudiantes desempeña alguna actividad laboral.

Con respecto a los factores del entorno familiar, se observa que el $83 \%$ de los estudiantes habita en el área urbana. Además, el 
95\% proviene de la provincia de Pichincha ${ }^{4}$, donde se ubica la capital del Ecuador, Quito, y la sede de la universidad analizada. Los estudiantes de las provincias de Imbabura y Tungurahua (cercanas a Pichincha) representan el $1 \%$ y el resto se reparte entre las otras provincias del país. En lo relacionado con el ingreso per cápita de los estudiantes, para facilitar la observación de los datos obtenidos, se agrupó a la variable en quintiles de ingreso. La carrera que tiene más representación de los quintiles más bajos es MA, mientras que la carrera IC tiene el porcentaje más bajo de personas pertenecientes al quintil 1 (ver Tabla 4). Una variable relacionada con la situación económica es el nivel de escolaridad de los padres. Según las respuestas de los encuestados, $43 \%$ de los padres y $34 \%$ de las madres tienen un título de educación superior (tercer y cuarto nivel); adicionalmente, mientras que en las carreras de IC, IM y MA la mayor parte de estudiantes tienen padres que no tienen estudios universitarios.

Tabla 4. Distribución de los estudiantes por quintil de ingreso

\begin{tabular}{ccccc} 
Quintil & FI & IC & IM & MA \\
\hline 1 & $18 \%$ & $16 \%$ & $22 \%$ & $27 \%$ \\
2 & $27 \%$ & $25 \%$ & $16 \%$ & $18 \%$ \\
3 & $12 \%$ & $24 \%$ & $19 \%$ & $21 \%$ \\
4 & $22 \%$ & $20 \%$ & $21 \%$ & $12 \%$ \\
5 & $20 \%$ & $15 \%$ & $22 \%$ & $21 \%$ \\
\hline
\end{tabular}

Una variable relevante para el análisis del desempeño de los estudiantes universitarios constituye el sistema de ingreso a la universidad. No obstante, el bajo porcentaje de estudiantes que ingresaron a la universidad con el sistema antiguo en la muestra $(5 \%)$, no permitió realizar un análisis comparativo más detallado. De todas formas, en la estimación se incluyó una variable dicotómica para diferenciar el sistema de ingreso de los estudiantes.

\subsection{Estimación del modelo econométrico}

Inicialmente, en la Tabla 5 se presenta una tabla que contiene la estadística descriptiva de algunas variables incluidas en el modelo econométrico que tiene por objetivo determinar los factores en el IRA estudiantil universitario.

Por otra parte, los resultados de la estimación se muestran en la Tabla 6. Debido a la presencia de heteroscedasticidad se realizó la estimación considerando errores robustos (ver Anexo 1). El análisis de multicolinealidad no reportó la presencia de variables relacionadas. No obstante, el análisis de variable omitida sugiere la presencia de este problema (ver Anexo 2). La consecuencia de omitir una variable relevante es el sesgo de los estimadores (Greene, 2004), es decir, los signos de las variables podrían ser opuestos a lo esperado según la teoría económica y la evidencia empírica previa. A pesar de que algunas variables individuales, como el IQ, la nota de ingreso a la universidad, la condición cognitiva, al igual que ciertas variables del contexto institucional pueden afectar a los resultados, la no disponibilidad de estas variables no permitió colocarlas en el modelo. Con todo, como se describe a continuación, los resultados muestran un comportamiento coherente con los resultados obtenidos en investigaciones previas, lo cual es un indicativo de que la omisión de ciertas variables no podría comprometer a las estimaciones.

Tabla 5. Estadística descriptiva de las variables

\begin{tabular}{lcccc}
\hline \multicolumn{1}{c}{ Variable } & Media & $\begin{array}{c}\text { Desviación } \\
\text { estándar }\end{array}$ & Mín. & Máx. \\
\hline $\begin{array}{l}\text { Créditos aprobados } \\
\text { Edad }\end{array}$ & 102 & 70 & 0 & 234 \\
\hline
\end{tabular}

Como se puede observar en la Tabla 6, al igual que lo mencionado por Guzmán (2012) y Ramírez (2014), la edad se muestra como un determinante que afecta negativamente al IRA y podría deberse a que en la universidad analizada generalmente una edad superior a la deseada en cada nivel cursado implica que se ha reprobado alguna o algunas materias. De hecho, la variable reprobó es significativa y negativa, lo cual es un resultado esperado debido al método de cálculo del IRA (descrito en la sección 5.1.1). Por otro lado, el efecto significativo y positivo del número de créditos aprobados sugiere que los estudiantes con una mayor experiencia en la universidad muestran un mejor rendimiento académico.

Tabla 6. Factores que afectan al desempeño de los estudiantes

\begin{tabular}{|c|c|}
\hline Variable & \\
\hline \multirow[t]{2}{*}{ Edad } & -0.0293 \\
\hline & $(0.0002)$ \\
\hline \multirow[t]{2}{*}{ Reprobó } & -0.1900 \\
\hline & $(0.0000)$ \\
\hline \multirow[t]{2}{*}{ Ln(créditos) } & 0.2690 \\
\hline & $(0.0000)$ \\
\hline \multirow[t]{2}{*}{ FI } & -0.1510 \\
\hline & -0.0001 \\
\hline \multirow[t]{2}{*}{ MA } & -0.1260 \\
\hline & $(0.0260)$ \\
\hline \multirow[t]{2}{*}{$\mathrm{IM}$} & -0.0761 \\
\hline & $(0.0095)$ \\
\hline \multirow[t]{2}{*}{ BGU } & 0.0581 \\
\hline & $(0.1233)$ \\
\hline \multirow[t]{2}{*}{ Técnico } & $0.145^{* *}$ \\
\hline & $(0.0054)$ \\
\hline \multirow[t]{2}{*}{ Educación superior padre } & -0.00198 \\
\hline & $(0.9512)$ \\
\hline \multirow[t]{2}{*}{ Educación superior madre } & 0.0634 \\
\hline & $(0.0441)$ \\
\hline \multirow[t]{2}{*}{ Quintil 2} & 0.1090 \\
\hline & $(0.0212)$ \\
\hline \multirow[t]{2}{*}{ Quintil 3} & 0.0496 \\
\hline & $(0.2279)$ \\
\hline \multirow[t]{2}{*}{ Quintil 4} & 0.0612 \\
\hline & $(0.1776)$ \\
\hline \multirow[t]{2}{*}{ Quintil 5} & 0.0308 \\
\hline & $(0.5021)$ \\
\hline \multirow[t]{2}{*}{ Constante } & 2.768 \\
\hline & $(0.0000)$ \\
\hline $\mathrm{R}^{2}$ & 0.5227 \\
\hline
\end{tabular}

${ }^{4}$ Una provincia es una división administrativa territorial en que se organizan algunos estados; este es el caso de Ecuador. 
El tipo de especialidad cursada en el colegio, también se muestra importante en la determinación del IRA al sugerir que estudiantes que cursaron un Bachillerato Técnico tendrían un mejor desempeño que los estudiantes del Bachillerato en Ciencias; esto, sin embargo, se contrapone a los hallazgos de la literatura previa, puesto que se esperaría que las mejores calificaciones en las carreras analizadas sean obtenidas por estudiantes cuyas carreras de secundaria se centran en ciencias exactas (Lerner, 2012). Esta inconsistencia podría deberse a que desde el 2008 se realizaron cambios importantes en la categorización y estructura de las carreras del bachillerato por parte del gobierno; cambios que no se ejecutaron de manera simultánea en todas las instituciones de educación.

El tipo de carrera elegida resulta ser también un factor importante en el IRA; pues en el modelo estimado se puede observar que los estudiantes de las carreras FI, MA e IM tienen un desempeño menor que los de la carrera IC.

Con respecto al entorno familiar, se observa que los estudiantes del quintil 2 tienen un rendimiento superior al de los estudiantes del quintil 1. Esto puede deberse a que la situación económica bien podría favorecer no solo el acceso de los jóvenes provenientes de los estratos socioeconómicos superiores (Jerrim, Chmielewski \& Parker, 2015), sino que también este aspecto podría coadyuvar a que el estudiante curse sus estudios en mejores condiciones que los estudiantes que sortean dificultades económicas (Mountford-Zimdars, Moore y Graham, 2016). Finalmente, el hecho de que la madre del estudiante tenga un título universitario también incide positivamente sobre el IRA. Este resultado ha sido reportado por hallazgos previos (Garbanzo, 2007).

\section{CONCLUSIONES}

Este artículo tiene por objetivos caracterizar socioeconómicamente al alumnado de una facultad de una universidad pública ecuatoriana y determinar los factores que influyen en el desempeño académico de tal grupo. A partir de los resultados, se puede concluir que la mayor parte de los estudiantes de la universidad analizada son; mestizos, provienen de colegios públicos, habitan en el área urbana de Pichincha y la carrera que están estudiando en el momento de la encuesta fue la que consideraron como primera prioridad cuando tuvieron que elegir la carrera en el SNNA. Existe un mayor porcentaje de hombres, con excepción de la carrera de IC, y la gran mayoría de los encuestados ha reprobado alguna vez una materia. Debido a la composición de la muestra, no fue posible determinar si existen diferencias importantes entre el IRA de los estudiantes que ingresaron mediante el SNNA y aquellos que lo hicieron mediante el sistema anterior. Con respecto a las variables que influyen en el desempeño académico, en el análisis se incluyeron variables que corresponden a factores individuales, sociales e institucionales y todas las dimensiones parecerían afectar al rendimiento académico, sugiriendo una interrelación entre éstas. Los resultados de este estudio contribuyen al debate en torno a los cuestionamientos del SNNA y sus objetivos de eficiencia y equidad y sugieren que la proporción de estudiantes pertenecientes a sectores menos aventajados continúa siendo casi nula y, que la mayor parte de estudiantes estaría cursando la carrera de su preferencia y que se sentiría satisfecho con esta. A la luz de estos resultados, se hace necesario que los formuladores de política pública realicen un ejercicio para repensar el sistema de educación superior actual y se apueste seriamente en un proceso de inclusión de los excluidos de siempre. Como recomendación, estudios posteriores a este podrían incluir aspectos relacionados con los docentes, así como medidas que reflejen el ambiente institucional con más precisión.

\section{REFERENCIAS}

Baena, M. D. (1999). El papel de la educación superior en el crecimiento y desarrollo de los países iberoamericanos. SCRIPTA NOVA Revista Electrónica de Geografía y Ciencias Sociales, 3, 1.

Chiroleu, A. (2011). La educación superior en América Latina: ¿Problemas insolubles o recetas inadecuadas? Universidade de Sorocaba, 16(3), 631-653.

Cortez, F., Tutiven, J. \& Villavicencio, M. (2017). Determinantes del Rendimiento Académico Universitario. Revista Publicando, 10(1), 284-296.

Erazo, O. (2012). El rendimiento académico, un fenómeno de múltiples relaciones y complejidades. Revista Vanguardia Psicológica Clínica Teórica y Práctica, 2(2), 144-173.

Fonseca, G. \& García, F. (2016). Permanencia y abandono de estudios en estudiantes universitarios: un análisis desde la teoría organizacionalPermanence and dropout rates among university students: An organizational theory analysis. Revista de la Educación Superior, 45(179), 25-39. http://dx.doi.org/10.1016/j.resu.2016.06.004

Garbanzo, C. (2007). Factores asociados al rendimiento académico en estudiantes universitarios, una reflexión desde la calidad de la educación superior pública. Revista Educación, 31(1), 43-63.

Greene, W. (2004). Análisis econométrico. Quinta Edición. Madrid: Prentice. Hall.

Guzmán, M. (2012). Modelos predictivos y explicativos del rendimiento académico. Tesis Doctoral. Universidad Complutense de Madrid. Madrid, España.

Jerrim, J., Chmielewski, A. \& Parker, P. (2015). Socioeconomic inequality in access to high-status colleges: A cross-country comparison. Research in Social Stratification and Mobility, 42, 20-32. https://doi.org/10.1016/j.rssm.2015.06.003

Larrea, C. (2006). Universidad, investigación científica y desarrollo en América Latina y Ecuador. Ponencia presentada ante el congreso "Universidad y Cooperación para el Desarrollo" en la Universidad Complutense de Madrid. Abril, 2006. 
Lerner, J. (2012). Rendimiento académico de los estudiantes de pregrado de la universidad EAFIT. Cuadernos de Investigación Universidad EAFIT.

Leyton, D., Sánchez, C. \& Ugalde, P. (2010). Estudio percepción de los jóvenes sobre la ciencia y profesiones científicas. Santiago.

Li, I. \& Carroll, D. (2017). Factors influencing university student satisfaction, dropout and academic performance: An Australian Higher Education Equity Perspective. . National Centre for Students Equity Higher Education (NCSEHE).

Martínez, C. (2011). Factores relacionados con el rendimiento académico en la asignatura Morfofisiología Humana IV, del Programa Nacional de Formación de Medicina Integral Comunitaria. Revista Médica Electrónica, 33(2).

Martínez, J. \& Salazar, R. (2014). Factores determinantes sobre el rendimiento académico en estudiantes de las facultades de medicina, enfermería, microbiología, psicología, arquitectura y derecho de la PUCE primer semestre de la carrera universitaria, en el período agosto-diciembre 2013. Quito.

Mountford-Zimdars, A., Moore, J. \& Graham, J. (2016). Is contextualised admission the answer to the access challenge? Perspectives: Policy and Practice in Higher Education, 20(4), 143-150. DOI: 10.1080/13603108.2016.1203369

Ponce, J. \& Carrasco, F. (2016). Acceso y equidad a la educación superior y posgrado en el Ecuador, un enfoque descriptivo. Revista Latinoamericana de Políticas y Acción Pública, 3(2), 9-22.

Psaharopoulos, G. (1994). Returns to investment in education: A global update. World development, 22(9), pp. $1325-1343$

Ramírez, C. (2014). Factores asociados al desempeño académico según nivel de formación pregrado y género de los estudiantes de educación superior Colombia. Revista Colombiana de Educación(66), 203-224.

Scheaffer, R., Mendenhall, W. \& Ott, L. (2006). Elementos de muestreo. Florida, United States: Editorial Paraninfo.

Shahzadi, E. \& Ahmad, Z. (2011). A study on academic performance of university students. Eighth International Conference on Recent Advances in Statistics, 255-268. Lahore.

Sibanda, L., Gervase, C. \& Benedict, H. (2015). Factors influencing academic performance of university students. Demography and Social Economy, 103115. DOI: $10.15407 / \mathrm{dse} 2015.02 .103$
Sulemana, M., Bin, I. \& Majid, R. (2014). The perspective of Students on Factors Affecting Their Academic Performance at the Tertiary Level. British Journal of Education, Society \& Behavioural Science, 4(8), 1021-1028. https://doi.org/10.9734/BJESBS/2014/8802

Valero, A. \& Van Reenen, J. (2019). The economic impact of universities: Evidence from across the globe. Economics of Education Review, 68, 53-67. https://doi.org/10.1016/j.econedurev.2018.09.001

Wimshurst, K., Wortley, R., Bates, M. \& Allard, T. (2006). The impact of institutional factors on student academic results: implications for 'quality' in universities. Higher Education Research \& Development, $25 . \quad$ DOI: $10.1080 / 07294360600610370$

Wooldridge, J. (2012). Introductory Econometrics: A modern approach. Michigan, United States: SOUTHWESTERN Cengage Learning.

\section{BIOGRAFÍAS}

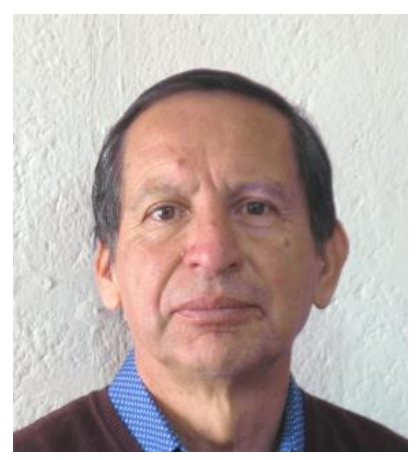

Julio Medina. Matemático formado en la Escuela Politécnica Nacional (EPN) de Quito-Ecuador. Master y Doctor en Matemáticas Aplicadas de la Universidad de Grenoble Alpes-Francia. Actualmente Profesor del Departamento de Economía Cuantitativa (DEC) de la EPN. Coordinador de la Maestría en Investigación Operativa y Logística de la EPN, y de las carreras de Ingeniería en Ciencias Económicas y Financieras de la EPN. Fue Profesor invitado a la Escuela Politécnica Federal de Lausana (EPFL)-Suiza. Inicialmente las líneas de investigación estuvieron relacionadas con Optimización matemática y Aproximación para continuar con Métodos variacionales para Ecuaciones en Derivadas Parciales. Premio a la mejor investigación de la EPN (1987). Actualmente miembro del equipo asociado a la línea de investigación en Economía del DEC. 


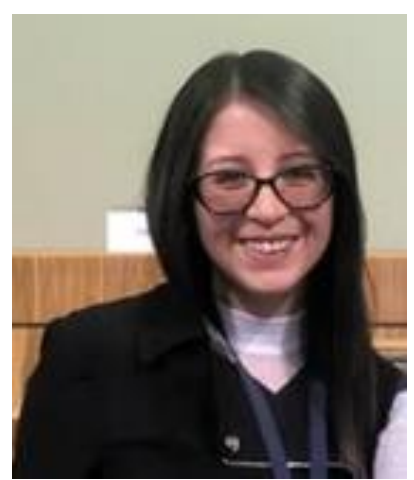

Kathia Pinzón. Economista ecuatoriana. Actualmente es Investigadora Asociada del Centro de Estudios para el Desarrollo y Economía Aplicada (CEDEA) de FLACSO-Ecuador, consultora independiente (nacional e internacional). Recientemente culminó sus estudios de maestría en Economía del Desarrollo por FLACSO-Ecuador. Ha sido Técnico Docente e investigadora del Departamento de Economía Cuantitativa de la Escuela Politécnica Nacional. También se ha desempeñado como analista de información en la ex Secretaría Nacional de Planificación y Desarrollo, y en el Banco Central del Ecuador. Principales líneas de investigación: economía del desarrollo, dinámica productiva y laboral, economía política y del comportamiento, estadística y econometría, modelización teórica matemática.

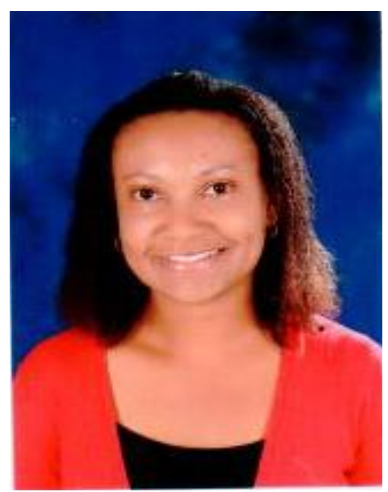

Yasmín Salazar Méndez. Desde 2015, es profesora titular de Econometría del Departamento de Economía Cuantitativa de la Facultad de Ciencias de la Escuela Politécnica Nacional. Doctora en Economía por la Universidad Federal Fluminense - Brasil (2015). Obtuvo una maestría en Ingeniería de Producción en la misma universidad (2008). Ingeniera Matemática graduada en la Escuela Politécnica Nacional (2003). Su investigación se centra en métodos cuantitativos y en la economía del bienestar, con énfasis en la desigualdad económica en América Latina.

\section{ANEXOS}

\section{Anexo 1}

Prueba para heteroscedasticidad - Breusch-Pagan Ho: Varianza constante

$\operatorname{chi} 2(1)=85.20$

Prob $>$ chi $2=0.0000$

\section{Anexo 2}

Prueba de variable omitida - Ramsey RESET Ho: el modelo no tiene variable omitida $\mathrm{F}(3,244)=23.81$

Prob $>\mathrm{F}=0.0000$ 